\title{
Characterization of the cytochrome P450 monooxygenase genes (P450ome) from the carotenogenic yeast Xanthophyllomyces dendrorhous
}

Pamela Córdova', Ana-María Gonzalez', David R. Nelson², María-Soledad Gutiérrez', Marcelo Baeza', Víctor Cifuentes ${ }^{1}$ and Jennifer Alcaíno ${ }^{1 *}$

\begin{abstract}
Background: The cytochromes P450 (P450s) are a large superfamily of heme-containing monooxygenases involved in the oxidative metabolism of an enormous diversity of substrates. These enzymes require electrons for their activity, and the electrons are supplied by NAD(P)H through a P450 electron donor system, which is generally a cytochrome P450 reductase (CPR). The yeast Xanthophyllomyces dendrorhous has evolved an exclusive P450-CPR system that specializes in the synthesis of astaxanthin, a carotenoid with commercial potential. For this reason, the aim of this work was to identify and characterize other potential P450 genes in the genome of this yeast using a bioinformatic approach.

Results: Thirteen potential P450-encoding genes were identified, and the analysis of their deduced proteins allowed them to be classified in ten different families: CYP51, CYP61, CYP5139 (with three members), CYP549A, CYP5491, CYP5492 (with two members), CYP5493, CYP53, CYP5494 and CYP5495. Structural analyses of the X. dendrorhous P450 proteins showed that all of them have a predicted transmembrane region at their $\mathrm{N}$-terminus and have the conserved domains characteristic of the P450s, including the heme-binding region ( $F x x G \times R \times C x G$ ); the PER domain, with the characteristic signature for fungi (PxRW); the ExxR motif in the K-helix region and the oxygenbinding domain (OBD) (AGXDTT); also, the characteristic secondary structure elements of all the P450 proteins were identified. The possible functions of these P450s include primary, secondary and xenobiotic metabolism reactions such as sterol biosynthesis, carotenoid synthesis and aromatic compound degradation.

Conclusions: The carotenogenic yeast $X$. dendrorhous has thirteen P450-encoding genes having potential functions in primary, secondary and xenobiotic metabolism reactions, including some genes of great interest for fatty acid hydroxylation and aromatic compound degradation. These findings established a basis for future studies about the role of P450s in the carotenogenic yeast $X$. dendrorhous and their potential biotechnological applications.
\end{abstract}

Keywords: Cytochrome P450, Xanthophyllomyces dendrorhous, Astaxanthin synthase

\footnotetext{
* Correspondence: jalcainog@u.uchile.cl

${ }^{1}$ Departamento de Ciencias Ecológicas y Centro de Biotecnología, Facultad de Ciencias, Universidad de Chile, Las Palmeras 3425, Casilla 653, Santiago, Chile

Full list of author information is available at the end of the article
} 


\section{Background}

Cytochrome P450 enzymes (P450s or CYPs) constitute a large superfamily of heme containing monooxygenases widely distributed in different organisms from all the domains of life, including animals, plants, fungi and prokaryotes [1]. These enzymes catalyze regio- and stereospecific conversions involved in the oxidative metabolism of a wide range of exogenous and endogenous substrates [2]. They are involved in the biosynthesis of many physiologically important compounds such as sterols, steroid hormones, fatty acids and vitamins [3]. Additionally, they participate in the biosynthesis of a vast array of secondary metabolites in plants, insects and fungi [4], providing them with adaptive advantages for the colonization of specific ecological and/or nutritional niches [5]. Moreover, these enzymes are involved in the detoxification of many xenobiotics such as drugs, pesticides, carcinogens and environmentally contaminating chemicals $[3,6]$. The typical reaction catalyzed by these enzymes is: $\mathrm{RH}+\mathrm{O}_{2}+2 \mathrm{e}^{-}+2 \mathrm{H}^{+} \rightarrow \mathrm{ROH}+\mathrm{H}_{2} \mathrm{O}$, where the electrons are transferred from $\mathrm{NAD}(\mathrm{P}) \mathrm{H}$ to $\mathrm{P} 450$ s by an electron donor [7].

P450 monooxygenase systems have been categorized into 10 classes depending on the proteins participating in the electron transfer [8]. Enzymes representatives of classes II, VIII and IX have been identified in Fungi [9], with class II being the most common class of P450s found in eukaryotic organisms.

Class II eukaryotic P450s are located in the endoplasmic reticulum, and the electron donor is usually the diflavin cytochrome P450 reductase (CPR), which contains FAD and FMN. The electron flow passes from NADPH to FAD to FMN and finally to P450, giving the specificity of the reaction [10, 11]. In most organisms, only one CPR-encoding gene exists, indicating that CPR can reduce many different $\mathrm{P} 450 \mathrm{~s}$ in a single species. Accordingly, Lah and co-workers [12], through an extensive in silico analysis of several fungal genomes, were able to identify between 20 and 155 putative P450encoding genes among the analyzed filamentous fungi. However, the number of P450-encoding genes found in yeasts in general was lower, ranging from 2 in Schizosaccharomyces pombe to 19 in Candida albicans. It has been proposed that a high number of P450s is related to a filamentous growth form in the context of occupying diverse ecological niches [12].

Currently, more than 19,000 P450s have been included in P450 databases and almost 8000 have been named according to the standard P450 nomenclature; this number is continually growing as new genome sequences become available $[5,13,14]$. One of the main reasons for the huge interest in identifying P450s genes from different organisms is their participation in the synthesis of secondary metabolites of biomedical, agricultural and industrial interest [15]. Along these lines, a P450 system has been found to be involved in the last step of the carotenogenesis pathway in the yeast Xanthophyllomyces dendrorhous, leading to astaxanthin production [16-18]. Unlike other astaxanthin-producing organisms, X. dendrorhous has a single astaxanthin synthase (CrtS, encoded by the $c r t S$ gene), which is related to a $3 \mathrm{~A}$ subfamily member cytochrome $\mathrm{P} 450$ in vertebrates $[16,17]$ and to the CYP64 clan in fungi, and catalyzes the hydroxylation and ketolation that convert beta-carotene to astaxanthin. This point suggests that in this yeast only, a unique P450 system has developed and specialized in the synthesis of astaxanthin, as $X$. dendrorhous is the only known organism to date that can synthesize astaxanthin from betacarotene through a P450 system. Furthermore, the crtR gene from $X$. dendrorhous, which encodes a CPR-type enzyme (CrtR), was characterized, and it was proved to be essential for the synthesis of astaxanthin [18]. Additionally, Ukibe and co-workers stated that " $X$. dendrorhous CrtS is a unique cytochrome P450 protein that has high specificity for its own P450 reductase", as in metabolically engineered $S$. cerevisiae strains with the $X$. dendrorhous carotenogenic genes, astaxanthin production, even in low amounts, was only achieved when CrtS was coexpressed with CrtR [19]. This point suggests that CrtS and CrtR have evolved together, specializing in astaxanthin formation. In addition to astaxanthin synthase, two other cytochrome P450-encoding genes from $X$. dendrorhous have been functionally described: CYP61 [20] and CYP51 [21], both involved in ergosterol biosynthesis.

It must to be considered that sedentary organisms, such as fungi and plants, depend on their overall metabolism to meet their environmental conditions and that they have developed complex biochemistries to defend themselves; cytochrome P450 enzymes have proven to play a key role in such processes [12, 22]. Indeed, a protective role against oxidative stress has already been attributed to astaxanthin biosynthesis in $X$. dendrorhous (in which a cytochrome P450 is involved); thus, it is conceivable to expect that this yeast has developed other defense mechanisms that could also include cytochrome P450s (for example, xenobiotics degradation or the synthesis of secondary metabolites besides astaxanthin). The great biotechnological impact of $X$. dendrorhous and the uniqueness of the CrtS-CrtR cytochrome P450 system make this yeast an attractive model to investigate alternative cytochrome P450 redox systems. Taking this into account along with the unquestionable importance of these systems in the metabolism of all organisms and the paucity of knowledge about them in $X$. dendrorhous, this work aimed to provide a detailed genome-wide structural, genetic and phylogenetic characterization of the P450ome of this yeast. 


\section{Methods}

\section{Strain and growth conditions}

The wild-type UCD 67-385 (ATCC 24230) X. dendrorhous strain was used for genome and transcriptome sequencing. The yeast was grown at $22{ }^{\circ} \mathrm{C}$ with constant agitation in YM rich medium (1\% glucose, $0.3 \%$ yeast extract, $0.3 \%$ malt extract and $0.5 \%$ peptone). Alternatively, it was grown in minimal medium $\left(\mathrm{MM}_{\mathrm{V}}\right)$ supplemented with $2 \%$ glucose [23] or $2 \%$ succinate [24] as the carbon source.

\section{Nucleic acid extraction, genome and transcriptome sequencing}

DNA was extracted from protoplasts according to [25], and a yield of chromosomal DNA greater than $50 \mathrm{~kb}$ was obtained.

Total RNA extraction was performed according to a modified protocol [26] of Chomczynski and Sacchi [27]. Briefly, total RNA was extracted from cell pellets broken through mechanical rupture with $0.5 \mathrm{~mm}$ glass beads (BioSpec, Bartlesville, OK, USA) and shaking in a vortex apparatus for $10 \mathrm{~min}$, followed by the addition of TriReagent (AmbionTM, Thermo Fisher Scientific Inc., Waltham, MA, USA). The lysate was incubated for $10 \mathrm{~min}$ at room temperature, and $150 \mu \mathrm{l}$ of chloroform per $\mathrm{ml}$ of Tri-Reagent used was added, mixed, centrifuged for $5 \mathrm{~min}$ at $4000 \mathrm{x} \mathrm{g}$, after which the aqueous phase was recovered. Then, two consecutive extractions with acidic phenol:chloroform (1:1) were done, and the RNA was precipitated by incubation at room temperature for $10 \mathrm{~min}$ with two volumes of isopropanol. The RNA was washed with $75 \%$ ethanol, suspended in RNase-free $\mathrm{H}_{2} \mathrm{O}$ and quantified spectrophotometrically at $260 \mathrm{~nm}$ in a V-630 UV-Vis Spectrophotometer from JASCO (JASCO, Easton, MD, USA), according to Sambrook et al. [28].

Genomic and transcriptomic sequencing was conducted as described previously [29]. For transcriptomic analyses, total RNA was extracted from yeast cultures at the early exponential $(\sim 18 \mathrm{~h})$ and initial stationary $(\sim 72 \mathrm{~h})$ phases of growth, grown in minimal media supplemented either with glucose or succinate as the sole carbon source, generating a total of four different conditions (G18, G72, S18 and S72: Glucose or Succinate at 18 or $72 \mathrm{~h}$ of culture) representing two types of metabolism in $X$. dendrorhous (fermentative in glucose and aerobic in succinate) to allow a better analysis of gene expression in different conditions. The assembly and analysis of the obtained transcriptomes were performed using the CLC Genomics Workbench 5 program.

\section{Identification and characterization of the putative P450- encoding genes}

The whole collection of contigs and scaffolds of the genome and transcriptomes was analyzed with the CLC Genomic Workbench software and compared by BLAST analysis against two P450 specialized databases: the David Nelson cytochrome P450 homepage web site (http://drnelson.uthsc.edu/CytochromeP450.html) [13] and the fungal cytochrome $\mathrm{P} 450$ database (http:// p450.riceblast.snu.ac.kr) [5]. In this way, the putative $X$. dendrorhous P450-encoding genes were identified. Gene sequences were analyzed with Geneious 10.0.2, and the analyses of the deduced protein sequences were performed using programs available online: Protein Calculator v3.3 (available at http://protcalc.sourceforge.net) was used to estimate protein parameters such as molecular weight and pI; TMpred (available at http://www.ch.embnet. org/software/TMPRED_form.html) [30] was used to predict transmembrane regions; the CYP450 Engineering database (available at https://cyped.biocatnet.de/) and the programs JPred (available at www.compbio.dundee.ac.uk/jpred/) [31] and SWISS-MODEL (available at https://swissmodel. expasy.org/) [32, 33] were used to predict secondary structure elements characteristic of P450 enzymes (the protein prediction servers were visited for the last time on March 28, 2017).

To search for cis-regulatory elements, the promoter region, defined as the 1500 nucleotides immediately upstream of the translation start codon of each P450encoding gene of $X$. dendrorhous, was analyzed with four programs: MATCH [34], PATCH (both available at http://gene-regulation.com/pub/programs.html), TFBIND (available at http://tfbind.hgc.jp/) [35] and JASPAR (available at http://jaspar.genereg.net/) [36]. The programs MATCH, PATCH and TFBIND use the TRANSFAC database, while JASPAR uses the JASPAR database (the cis-regulatory element websites were visited for the last time on March 28, 2017).

Phylogenetic analysis was carried out using Clustal Omega (available at http://www.ebi.ac.uk/Tools/msa/ clustalo) and Simple Phylogeny (available at http://www.ebi .ac.uk/Tools/phylogeny/simple_phylogeny) for protein multiple alignments and phylogenetic tree construction, respectively (the alignment and phylogenetic tree construction web sites were visited for the last time on March 28, 2017). For phylogenetic tree construction, the neighborjoining method was used, and the obtained tree was visualized with the software MEGA 6.0 [37].

The sequences of P450s from other organisms used in the construction of the phylogenetic tree were the following: S. cerevisiae: ERG11 [NP_011871.1], ERG5 [P54781.1]; Aspergillus nidulans: bzuA [AAL10516.1], ppoC [AAT36614.1], ppoA [AAR88626.1], ahbB [AAR 15377.1], stcF [AAC49196.1], stcB [AAC49196.1], phacA [CAB43093.1], phacB [ABB20530.1], ivoC [CBF77085.1], CYP62 [XP_681093.1]; Aspergillus nonius: cypA [AAS 90045.1]; Aspergillus fumigatus: CYP51a [ACF17705.1], CYP51b [AAK73660.1], gliC [EDP49542.1], gliF [AAW 03300.1], ftmE [BAH23999.1], ftmC [BAH23996.1], ftmG 
[BAH24001.1]; Aspergillus clavatus: CYP619C2 [ACG 60892.1], CYP619C3 [ACG60891.1]; Aspergillus oryzae: nicA [BAC01275.1]; Aspergillus niger: bph [P17549.1]; Aspergillus parasiticus: aflU [Q6UEH4.1], aflQ [AAS 66031.1]; Cryptococcus neoformans: ERG11 [AAF 35366.1]; Aspergillus flavus: CYP51Ap [EED56341.1], CYP51Bp [EED50354.1], AF115 [AAT65721.1]; Phanerochaete chrysosporium: CYP51 [ACI23621.1]; Coprinopsis cinereus: eln2 [BAA33717.1]; Fusarium graminearum: tri1 [AAQ02672.1], tri11 [BAC22120.1], tri4 [AAK 53584.1]; Fusarium sporotrichioides: tri4 [AAB72032.1]; Fusarium oxysporum: fum6 [ACB12553.1], CYP505 [BAA82526.1], CYP55 [P23295.]; Myrothecium roridum: tri4 [AAC49958.1]; Leptosphaeria maculans: sirB [AAS 92544.1], sirC [AAS92547.1], sirE [AAS92549.1]; Penicillium paxilli: paxP [AAK11528.1], paxQ [AAK11527.1]; Candida albicans: dit2 [CAK54651.1], alk8 [CAA 75058.1], CYP51 [XP_716761]; Candida maltosa: P450alk [CAA39367.1], CYP52A3-b [AAC60531.1]; Candida glabrata: CYP61 [KTB21909.1]; Chaetomium chiversii: radP [ACM42407.1]; Streptomyces venezuelae: piKC [AAC68886.1]; Nectria haematococca: PDAT9 [AAC01762.1]; Trichosporon cutaneum: P450nor [BAB 60855.1]; Gibberella intermedia: P450-4 [Q701P2.1], P450-1 [CAF31353.1]; Gibberella moniliformis: fum2 [AAN74815.2]; Heterobasidion irregulare: CYP56 [XP 009550156], CYP65 [ETW74855.1], CYP59 [ETW 81982.1], CYP60 [ETW77818.1], CYP62 [XP_009548 132.1], CYP68 [ETW81986.1]; Yarrowia lipolytica: $A L K 3$ [BAA31435.1]; ALK8 [BAA31440]; ALK5 [BAA31437.1]. The gene names are indicated, followed by the $\mathrm{P} 450$ protein sequence accession numbers (GenBank or NCBI reference sequence).

\section{Results and discussion}

\section{Identification and structural analysis of $X$. dendrorhous} P450-encoding genes

Regarding the P450 genes, the cytochrome P450 homepage website built by Dr. David Nelson has been available since 1995 [13], and in addition, a fungal specialized cytochrome P450 database has been available online since 2008 [5]. The assembled genome (available in our laboratory and also published by Sharma et al. (2015) [38]) and transcriptomes of $X$. dendrorhous were compared against these two databases with the BLAST tool, allowing us to identify several putative P450 genes. As a result, 13 potential cytochrome P450-encoding genes, including crtS, CYP51 and CYP61, were found in the $X$. dendrorhous genome and were uploaded to GenBank (Table 1). Notably, the $X$. dendrorhous P450ome would be one of the largest found in yeasts, since previous studies have shown that most yeasts (unicellular fungi) have fewer than ten cytochrome P450s, with a few exceptions such as Candida albicans (19 cytochrome
P450s) and Yarrowia lipolytica (17 cytochrome P450s), unlike filamentous fungi that have often higher numbers of P450s encoding genes [12, 22]. Accordingly, the 13 cytochrome P450s of $X$. dendrorhous were named following the prescriptions of the International P450 Nomenclature Committee, where they fell into 10 families, each containing only one member except for CYP5139 (with three members) and CYP5492 (with two members) (Table 1).

The sequence analyses of the genomic and cDNA versions of each P450-encoding gene allowed us to determine their exon-intron structure and deduce the encoded polypeptide (Table 1). The structure of each gene was defined by sequence alignments, which were manually edited. The $X$. dendrorhous $\mathrm{P} 450$ genes have between 6 and 19 exons, ranging in size from as small as $4 \mathrm{bp}$ to $462 \mathrm{bp}$. The intronic sequences ranged from 72 to $354 \mathrm{bp}$ having the canonical GT-AG donor and acceptor splice site at their extremes; except for the fourth intron of CYP5490A1 and the first intron of CYP5492A2, which had the less common GC-AG splice sites. Additionally, the GT-CA splice sites were observed in the eleventh intron of CYP5139R1, which was confirmed by Sanger sequencing in several $X$. dendrorhous strains.

\section{Transcriptomic analysis of P450 gene expression}

To obtain more information about the P450 genes of $X$. dendrorhous, four transcriptomes of the wild-type strain UCD 67-385 were analyzed, and the level of each of the P450 gene transcripts was estimated by calculating its RPKM (reads per kilobase per million mapped reads) value, which mirrors the molar concentration of the transcript in the starting sample normalized to the length of the RNA and the total number of reads [39]. The analyzed transcriptomes were obtained from yeast cultures at the early exponential $(\sim 18 \mathrm{~h})$ and initial stationary $(\sim 72 \mathrm{~h})$ phases of growth, grown in minimal media supplemented either with glucose or succinate as the sole carbon source, generating a total of four different conditions (G18, G72, S18 and S72: Glucose or Succinate at $\mathbf{1 8}$ or $\mathbf{7 2} \mathrm{h}$ of culture) (Table 2). In general, the results show that the P450-encoding gene transcripts have similar RPKM values in the four studied conditions, but changes are observed in some cases such as the $\mathrm{crtS}$, CYP5491A1, CYP5492A1, CYP5493A1 and CYP5494A1 genes, whose RPKM values in the $G 72$ condition are higher than the values at the G18 condition, suggesting induction at late growth phases. This has also been observed in previous studies for the crtS gene [24].

On other hand, when the RPKM values of the S18 and S72 conditions were compared, no major changes were observed. Additionally, it was observed that the CYP5494A1 and CYP5492A2 transcript RPKM values were higher in the S18 condition than in G18, suggesting 


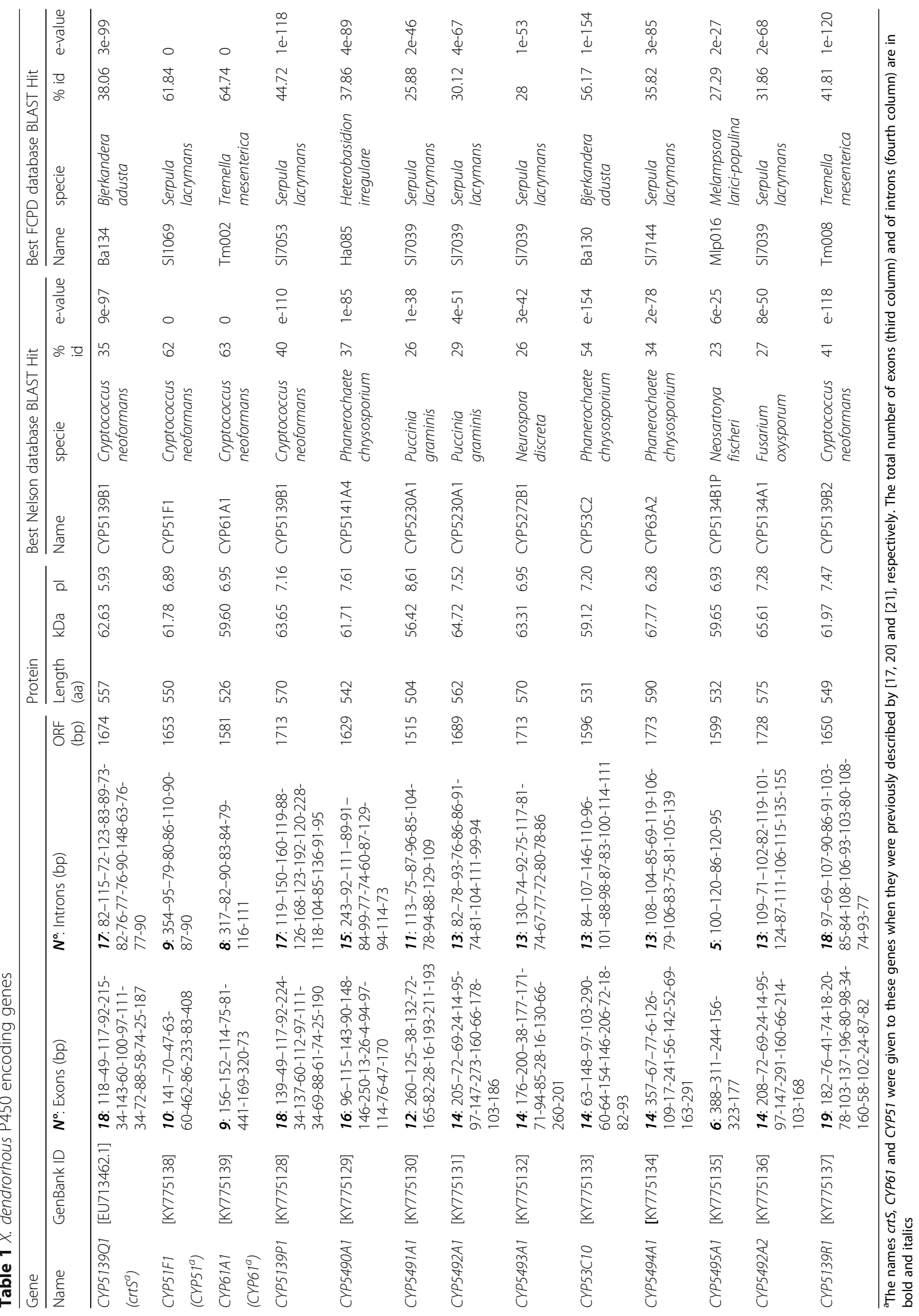


Table 2 RPKM values of the $X$. dendrorhous P450-encoding gene transcripts

\begin{tabular}{|c|c|c|c|c|c|c|c|}
\hline \multirow[b]{2}{*}{ Gene } & \multicolumn{4}{|c|}{ Condition $^{\mathrm{a}}$} & \multicolumn{3}{|c|}{ Normalization by G18 } \\
\hline & G18 & $\mathrm{G} 72$ & $\mathrm{~S} 18$ & $\mathrm{~S} 72$ & G72/G18 & S18/G18 & $\mathrm{S} 72 / \mathrm{G} 18$ \\
\hline crts & 85.4 & 596.3 & 87.9 & 245.2 & 7.0 & 1.0 & 2.9 \\
\hline CYP51 & 51.7 & 48.8 & 40.9 & 39.2 & 0.9 & 0.8 & 0.8 \\
\hline CYP61 & 110.9 & 133.0 & 51.7 & 41.7 & 1.2 & 0.5 & 0.4 \\
\hline CYP5139P1 & 27.4 & 57.0 & 64.0 & 67.4 & 2.1 & 2.3 & 2.5 \\
\hline CYP5490A1 & 20.1 & 23.4 & 19.0 & 44.8 & 1.2 & 0.9 & 2.2 \\
\hline CYP5491A1 & 7.8 & 31.7 & 5.4 & 11.4 & 4.1 & 0.7 & 1.5 \\
\hline CYP5492A1 & 4.6 & 180.2 & 11.8 & 31.1 & 39.4 & 2.6 & 6.8 \\
\hline CYP5493A1 & 11.4 & 32.9 & 7.8 & 16.9 & 2.9 & 0.7 & 1.5 \\
\hline CYP53C10 & 7.0 & 8.6 & 7.7 & 6.3 & 1.2 & 1.1 & 0.9 \\
\hline CYP5494A1 & 9.3 & 25.2 & 30.1 & 29.5 & 2.7 & 3.3 & 3.2 \\
\hline CYP5495A1 & 19.1 & 43.9 & 25.9 & 27.5 & 2.3 & 1.4 & 1.4 \\
\hline CYP5492A2 & 5.8 & 5.4 & 16.1 & 25.1 & 0.9 & 2.8 & 4.3 \\
\hline CYP5139R1 & 44.7 & 49.0 & 19.2 & 28.2 & 1.1 & 0.4 & 0.6 \\
\hline
\end{tabular}

${ }^{a}$ The four conditions $\mathrm{G} 18, \mathrm{G} 72, \mathrm{~S} 18$ and $\mathrm{S} 72$ were previously described in the text

differential expression depending on the carbon source or possibly related to the type of metabolism performed, either fermentative for growth on glucose or aerobic for succinate. As mentioned above, most of the P450 transcripts did not show major changes; this may be because some of the studied genes encode proteins involved in essential functions, such as CYP51 and CYP61. On the other hand, it is possible that some of the studied P450 genes are induced by an external factor; this may be the reason why no changes were observed in the studied conditions. An example of the latter occurs in Aspergillus niger, in which the cytochrome $\mathrm{P} 450$ benzoate-parahydroxylase gene is only induced when benzoate is added to the culture medium [40]. A similar phenomenon was observed in the yeast Candida tropicalis, which can metabolize phenol as a carbon source using a P450 system, where the cytochrome P450 content was induced by phenol [41].

It should be noted that among P450s, Cyp51 (lanosterol 14 alpha-demethylase) is the only widely conserved enzyme, having orthologs in organisms from bacteria to humans; this status suggests that the enzyme could represent the common ancestor of all P450s [42]. In addition, Cyp51 is the only enzyme in the P450 family that fulfills the same function in all biological kingdoms, namely, three-step sterol 14-demethylation in sterol biosynthesis [43]. In mammals, the biosynthesis of cholesterol is regulated by the SREBP (sterol regulatory element binding protein) pathway, which promotes the transcription of genes such as CYP51 in response to low cholesterol levels in the cell. This pathway has recently been characterized in the yeast $S$. pombe, and it was shown that the two P450 genes in this organism, CYP61 and CYP51, which are involved in ergosterol biosynthesis, are under the regulation of the SREBP pathway [44]. SREBP (named Sre1 in fungi) binds to SRE (sterol regulatory element) sequences at the promoter region of the target genes. To determine whether this transcriptional factor could be regulating P450-encoding genes in $X$. dendrorhous, the promoter region, comprising 1500 nucleotides upstream of the translation start codon of each gene, was bioinformatically analyzed. The SRE sequences identified by at least two of the programs used in the analyses are detailed in Table 3. According to the criteria used, it was possible to identify potential SRE sequences in most sequences except for those from the crtS, CYP5491A1 and CYP5494A1 genes, identifying possible SRE sites in the promoter region of $\mathrm{P} 450$ genes involved in ergosterol biosynthesis (CYP51 and CYP61) consistent with previous works. This result suggests that the majority of the P450s encoding genes from $X$. dendrorhous could be regulated, at least in part, by the same regulatory pathway as their potential common ancestor CYP51.

\section{Analysis of deduced amino acid sequences of $\mathrm{P} 450 \mathrm{~s}$}

The 13 potential cytochrome $\mathrm{P} 450$ gene sequences of $X$. dendrorhous were analyzed to find the corresponding ORFs, and the encoded proteins were deduced. The ORFs varied in length from 1296 to $1773 \mathrm{bp}$. The sizes of the deduced proteins ranged from 504 to 590 amino acids, consistent with the sizes of previously described P450 proteins $[45,46]$. The predicted molecular masses varied from 56.2 to $67.77 \mathrm{kDa}$, and the isoelectric points (pIs) ranged from 5.93 to 8.61 (Table 1).

Regarding the subcellular localization of the deduced P450 proteins, a putative hydrophobic transmembrane segment at the amino terminus was predicted by TMpred [30] analyses in all the deduced amino acid sequences. This finding is important as this feature allows class II P450 enzymes to anchor to the endoplasmic reticulum membrane [9].

Many studies have demonstrated that P450s share little sequence similarity, except for a few conserved domains needed to preserve their tertiary structure and enzymatic function [22]. The most conserved motif is the hemebinding region FxxGxRxCxG (also known as the $\mathrm{CxG}$ motif) containing the axial Cys ligand that binds to the heme. The motifs ExxR and PER form the E-R-R triad, which is important for locking the structure of the heme pocket in place and ensuring the stabilization of the core structure. Moreover, ExxR and FxxGxRxCxG contain the three most conserved amino acids in the cytochrome P450 protein family: the glutamic acid and arginine in the ExxR motif and the heme-binding cysteine in the CxG motif [47]. The last conserved motif, AGxDTT, contributes to oxygen binding and activation $[22,46]$. 
Table 3 SRE sequences detected in the promoter regions of $X$. dendrorhous P450-encoding genes

\begin{tabular}{|c|c|c|c|c|c|}
\hline \multirow{2}{*}{$\frac{\text { Gene }}{\text { CYP51 }}$} & \multirow{2}{*}{$\begin{array}{l}\text { Strand } \\
+\end{array}$} & \multirow{2}{*}{$\frac{\text { Sequence }\left(5^{\prime}-3^{\prime}\right)}{\text { GTGGGTCAC }}$} & \multicolumn{2}{|c|}{ Position (start-end) } & \multirow{2}{*}{$\begin{array}{l}\text { Program } \\
\text { JASPAR/TFBIND }\end{array}$} \\
\hline & & & -685 & -694 & \\
\hline & + & ATCACCTCTC & -95 & -104 & JASPAR/TFBIND \\
\hline \multirow[t]{2}{*}{ CYP61 } & + & ATCAACTGAC & -1412 & -1421 & JASPAR/TFBIND \\
\hline & + & ATCACCAGAG & -519 & -528 & JASPAR/TFBIND \\
\hline \multirow[t]{2}{*}{ CYP5139P1 } & + & CTCACACCAC & -1048 & -1057 & JASPAR/TFBIND \\
\hline & + & ATCACATGAT & -262 & -271 & JASPAR/TFBIND \\
\hline CYP5490A1 & + & GTTGGGTGAG & -709 & -718 & JASPAR/TFBIND \\
\hline \multirow[t]{3}{*}{ CYP5492A1 } & + & GTCATCTGAT & -1267 & -1276 & JASPAR/TFBIND \\
\hline & - & ATCACCCCAA & -367 & -376 & JASPAR/PATCH \\
\hline & + & ATCACGTCA & -314 & -323 & JASPAR/TFBIND \\
\hline CYP5493A1 & + & ATCAACCCAC & -413 & -422 & JASPAR/TFBIND/PATCH \\
\hline CYP53C10 & + & GTGACATGAT & -369 & -378 & JASPAR/TFBIND \\
\hline \multirow[t]{2}{*}{ CYP5495A1 } & + & GTGGGCTGAA & -1057 & -1066 & JASPAR/TFBIND \\
\hline & + & ATCACGTGAA & -102 & -111 & JASPAR/TFBIND/PATCH/MATCH \\
\hline \multirow[t]{2}{*}{ CYP5492A2 } & - & ATCACCTCAC & -985 & -994 & JASPAR/PATCH \\
\hline & - & ATCAAGCCAG & -522 & -531 & JASPAR/PATCH \\
\hline CYP5139R1 & + & GAGAGGTGAT & -313 & -322 & JASPAR/TFBIND \\
\hline
\end{tabular}

The ExxR and FxxGxRxCxG motifs are present in the 13 predicted $\mathrm{P} 450$ s from $X$. dendrorhous, and all of them have the three conserved amino acids (Fig. 1). Syed and Mashele performed an extensive comparative analysis of the P450 signature motifs among the fungal kingdom [47], and our results are in agreement with this previous work. Among the $X$. dendrorhous ExxR motifs of P450s, the ESLR pattern was the most abundant (in 4 sequences), followed by ETLR and EVLR (in 3 sequences each), EALR (in 2 sequences) and EILR (in 1 sequence).
Interesting, between the glutamic acid and the arginine at the first and last position of this motif, respectively, all the $X$. dendrorhous $\mathrm{P} 450$ s contain a leucine at the third position, which has also been shown to be the most predominant residue at this position in the fungal P450s. Serine, threonine and valine were shown to be the most frequent residues at the second position of the $X$. dendrorhous P450s ExxR motif, in agreement with what has been previously reported [47]. Each $X$. dendrorhous $\mathrm{P} 450$ showed a different FxxGxRxCxG

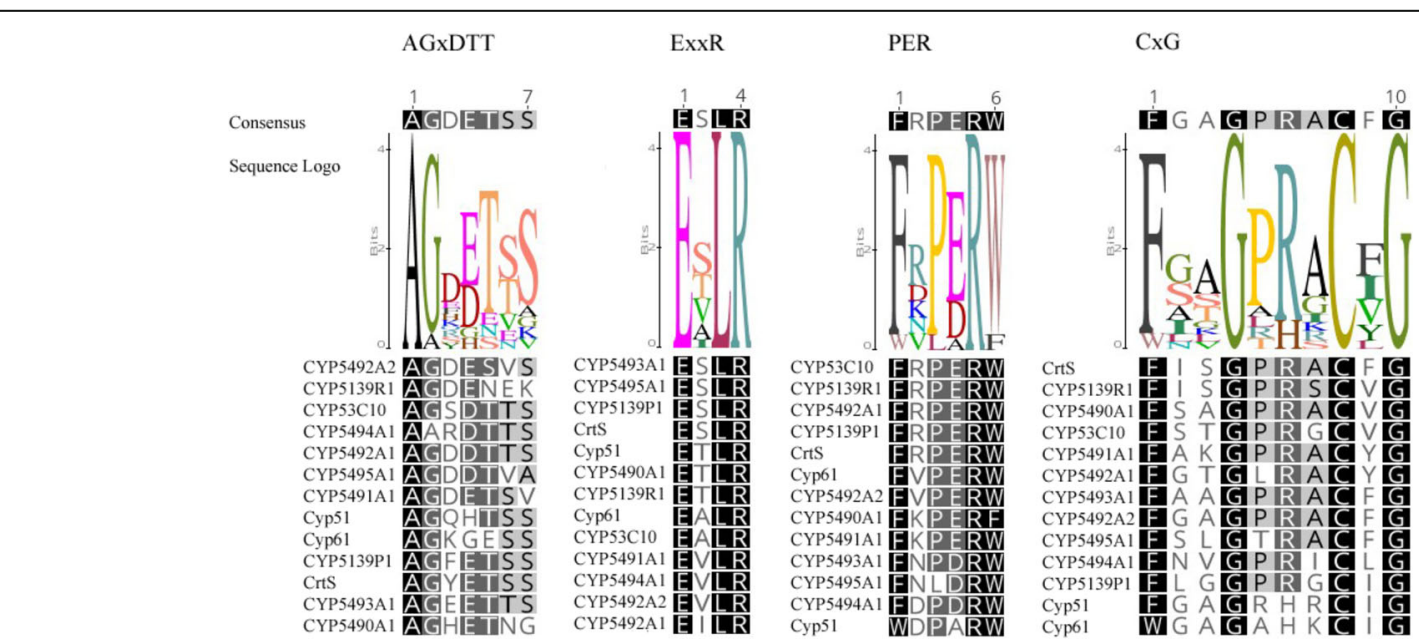

Fig. 1 Conserved motifs in the P450 proteins of $X$. dendrorhous. Multiple alignments of the conserved motifs AGXDTT, ExxR, PER and CXG of X. dendrorhous. Alignments are highlighted by percentage of identity as follows: black: $100 \%$ identity, gray: > $80 \%$ identity, light gray: $>60 \%$ identity and white: $<60 \%$ identity. Sequence logos show the conservation and relative frequency of each amino acid within each conserved motif. Alignments and sequence logos were constructed using the Geneious software 
motif pattern; however, all of them contained the conserved cysteine at the eighth position and had glycine at the fourth and tenth position. All the sequences had a phenylalanine at the first position, with the exception of Cyp61, which has a tryptophan. This last observation is not surprising since it has been shown that tryptophan is the second most frequent residue at this position among 47 fungal $\mathrm{P} 450$ sequences analyzed [22].

It has been reported that arginine is the predominant residue at the sixth position of the FxxGxRxCxG motif, and this is the case in all of the $X$. dendrorhous P450s except for Cyp51 and Cyp61, which have a histidine. In previous studies, this residue was shown to be the most common at this position in the Cyp61 family [47], the second most frequent among 47 fungal P450s analyzed and the most frequent among 105 bacterial P450s analyzed [22]. For the CxG pattern (the three last residues of the FxxGxRxCxG motif), four $X$. dendrorhous P450s have the CFG pattern, three each have a CIG and a CVG pattern, two have a CYG pattern and one has a CLG pattern. These results are different from what have been reported for this motif, as it was previously found that CPG was the most predominate triad in basidiomycetes, followed by CIG, CLG and CVG in second, third and fourth place, respectively. The most frequent pattern in $X$. dendrorhous (CFG) only represented $0.21 \%$ of a total of 4304 fungal P450 sequences and approximately 0.14\% of 2859 Basidiomycota P450 sequences analyzed previously [47]. Six of the 13 P450 proteins identified in $X$. dendrorhous (including $\mathrm{CrtS}$ ), have an aromatic aminoacid ( $\mathrm{F}$ or $\mathrm{Y}$ ) in the middle of the CxG triad, both uncommon residues at this position among fungal $\mathrm{P} 450 \mathrm{~s}$ (among the 4304 fungal P450 sequences previously analyzed, $0.21 \%$ and $0.09 \%$ were CFG and CYG, respectively) [47]. Although the effect of the different amino acids in the middle position of the triad CxG is still unknown, previous studies have demonstrated that different P450 families show preference for determined residues at this position suggesting that amino acids in this motif play a role in determining P450 structure, activity and substrate specificity [47]. Considering that the CrtS enzyme, which has the CFG triad, has the unique ability to convert $\beta$-carotene to astaxanthin by finally introducing both, a ketone and a hydroxyl group at both $\beta$-ionone rings of $\beta$-carotene [17], it is possible that the predominant CFG triad found in the P450s of $X$. dendrorhous contributed to a new substrate specificity related to the enzymes functions. Moreover, in a phylogenetic study of the $X$. dendrorhous P450s (see below), those having aromatics amino acids in the middle position of the CxG triad (CYP5491A1, CYP5492A1, CYP5492A2, CYP5493A1 and CYP5495A1) showed to be closely related, suggesting related functions.
The third conserved motif described as a P450 signature: PER [48], with the characteristic signature PxRW for fungi [46], was also found in the $13 \mathrm{X}$. dendrorhous P450s (Fig. 1). Although this motif has four amino acids, the two previous residues were also considered in the analysis, giving a six-amino-acid sequence, as a phenylalanine residue two positions before the conserved proline of the PxRW motif is highly conserved in most P450s [22]. Accordingly, in all but one of the $X$. dendrorhous P450s, a phenylalanine residue was found at the first position. As expected, in the third position, the most common residue was a proline, followed in sequence by a less conserved glutamic acid and a completely conserved arginine at the fifth position. Other than the conserved arginine, the most common amino acid was a tryptophan at the sixth position, consistent with previous descriptions of fungal P450s [22].

The last conserved motif, AGxDTT, also known as the oxygen binding domain (OBD), was found in all of the P450s of $X$. dendrorhous, but it was the least conserved in sequence, with the first (an alanine in all sequences), second (a glycine in most cases) and fifth (a threonine in most cases) positions being the most conserved ones (Fig. 1). At the fourth position, the most common residues were glutamic acid and aspartic acid, in agreement with other fungal P450s described [22, 46].

These results showed that all of the deduced gene products from all of the identified $X$. dendrorhous P450 genes have all the characteristic signature motifs described for this superfamily of proteins.

\section{P450 structure}

In the last few years, several studies have analyzed the structure of different $\mathrm{P} 450$ proteins. The increasing number of crystal structures published, together with comparative analyses of different P450 structures, have revealed that despite the low sequence identity $(10-30 \%)$, members of this superfamily of proteins have a conserved structural core [49]. In general, the secondary structure elements of these enzymes include at least four beta sheets ( $\beta 1$ to $\beta 4$ ) and 12 alpha helices ( $\alpha \mathrm{A}$ to $\alpha \mathrm{L})$. Also, in some structures, an additional beta sheet $(\beta 5)$ or insertions forming additional alpha helices (named B', C', J', $\mathrm{K}^{\prime}$ and $\mathrm{K}^{\prime \prime}$ ) have been found, with $\mathrm{K}^{\prime}$ and J' being the most frequent ones [50, 51]. Considering the above, the $X$. dendrorhous P450s proteins deduced using the CYP450 Engineering database (https://cyped.biocatnet.de) were analyzed. Using the "Standard Numbering" tool of this P450 structure database, positive results were obtained for proteins Cyp61, Cyp51, CrtS, Cyp5139P1, Cyp5490A1, Cyp53C10, Cyp5494A1 and Cyp5139R1 of X. dendrorhous; among them, the deduced secondary structures of the first two have been previously reported $[20,21]$. The SWISS- 
MODEL [32, 33] and JPred [31] servers available online were used to predict the secondary structure elements in the rest of the $X$. dendrorhous P450s (Cyp5491A1, Cyp5492A1, Cyp5493A1, Cyp5495A1 and Cyp5492A2). In this way, the characteristic regions known as the "Cys pocket" and "the meander loop" in P450 enzymes were identified in all of the deduced P450s of $X$. dendrorhous. The most conserved secondary structural elements $(\alpha \mathrm{A}-\alpha \mathrm{L}$ helices and $\beta 1-\beta 4$ sheets) according to [51] were all found in the predicted P450 proteins (Fig. 2 and Additional file 1). In Fig. 2, the predicted secondary structural elements of CrtS are shown as an example.

A less conserved structural element was $\beta 5$, which was not identified in four of the 13 P450s analyzed. Furthermore, the $\mathrm{B}^{\prime}$ and $\mathrm{C}^{\prime}$ alpha helices, which are not described as highly conserved in previous studies [50, 51], were present in ten of the $X$. dendrorhous P450s. In contrast, alpha helices $J^{\prime}$ and $K^{\prime}$ were found in all the predicted $\mathrm{P} 450$ proteins. Thus, the predicted secondary structure elements indicate that the proteins identified in this work have the characteristic secondary structural elements of P450 enzymes.

As previously mentioned, P450 enzymes require an electron donor partner. In $X$. dendrorhous, the CrtR protein, a CPR enzyme, plays that role and has been studied principally for its involvement in the last step of astaxanthin biosynthesis together with the CrtS enzyme (P450). Regarding this, a previous study [52] predicted which residues were important for the interfacial interaction between CrtS and CrtR. In the CrtS protein, eight residues were identified as implicated in the formation of salt bridges with CrtR: K98, K130, R131, R133, R134, K146, E320 and R499. Among them, only K130, R134,
K146 and E320 appeared to be specific for the CrtSCrtR interaction [52]. The sequences of all the $X$. dendrorhous P450s were analyzed to determine whether these eight residues are conserved. In CrtS, $\mathrm{K} 98$ is positioned between the helix $\alpha \mathrm{B}$ and $\beta$ strands $1-5$, and it is 32 residues away from the next conserved residue, K130, which is positioned in the helix $\alpha \mathrm{C}$. Taking this into account, residues potentially equivalent to CrtS K98 were found in ten of the 13 P450s analyzed (Fig. 2 and Additional file 1), and in the case of Cyp53C10, a histidine (H95) was found in this position, which would replace the function of the conserved arginine since both residues are positively charged amino acids. The next residues in CrtS that would be involved in the CrtS-CrtR interaction are grouped in the helix $\alpha \mathrm{C}$, comprising the region from position 130 to 134 (K130, R131, R133, R134). It must be noted that in this region in CrtS, five positively charged residues are in consecutive positions (although H132 would not be involved in formation of salt bridges as previously reported [52]), followed by K146 at helix $\alpha$ C, 11 positions later. The region from helix $\alpha \mathrm{C}$ to helix $\alpha \mathrm{D}$ of all $X$. dendrorhous P450s was screened for the presence of equivalent residues (Additional file 2). In general, at least three positively charged residues were found grouped inside the helix $\alpha \mathrm{C}$ except in the proteins Cyp51 and Cyp61, in which only a pair of positive residues were found. A residue potentially equivalent to K146 was found in all but one (Cyp5493A1) of the deduced proteins, replaced by an arginine in some cases (Additional file 2). Although it is difficult to ensure the equivalent correspondence since in some cases there was more than one possibility, residues potentially equivalent to $\mathrm{CrtS}$
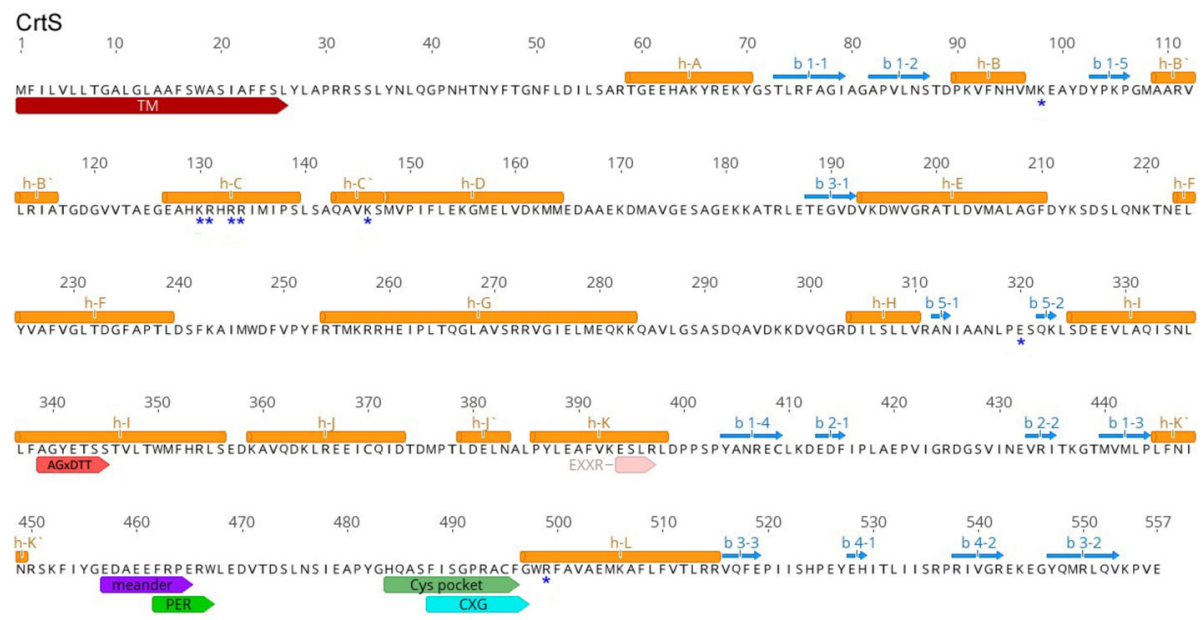

Fig. 2 Analysis of CrtS protein from X. dendrorhous. Characteristic conserved secondary elements of P450 proteins are shown along the CrtS protein. Alpha helices (h) and beta strands (b) are represented as orange cylinders and blue arrows, respectively. The transmembrane region (TM) and conserved regions such as the "meander loop" and the "Cys pocket" are also highlighted. The location of the conserved motifs AGXDTT, ExxR, PER and CXG are shown along the protein. Residues involved in the interaction CrtS-CrtR according to [52] are marked with asterisks 
E320 (positions between $\alpha \mathrm{H}$ and $\alpha \mathrm{I}$ ) were found in all of the $X$. dendrorhous $\mathrm{P} 450$ s proteins, including an aspartic acid in place of a glutamic acid in two cases. Finally, in the case of R499 from CrtS, which is positioned three residues after the conserved cysteine of the Cys pocket region, potentially equivalent residues were found in ten of the P450s, the arginine being replaced in some cases by a lysine and absent in Cyp51, Cyp61 and Cyp5495A1 (Additional file 1).

In general, the residues potentially involved in the interaction with the cytochrome $\mathrm{P} 450$ reductase $\mathrm{CrtR}$ are conserved among the $X$. dendrorhous P450s. Nevertheless, previous studies have demonstrated that a $X$. dendrorhous crt $^{-}$mutant is viable [18]; thus, P450s enzymes involved in essential processes such as ergosterol biosynthesis, as in the case of Cyp51 and Cyp61, should be able to interact with other electron donor partners such as the cytochrome b5 reductase/cytochrome b5 pathway [53], and it is possible that some of the salt-bridge-forming residues identified in the $\mathrm{CrtS}$ protein are not conserved in other P450s, because less specific interactions are needed compared to the CrtSCrtR interaction.

\section{Phylogenetic study}

As previously mentioned, the first analyses of the P450 sequences showed that the thirteen P450s from $X$. dendrorhous belong to 10 different protein families: CYP51, CYP61, CYP5139 (with three members), CYP549A, CYP5491, CYP5492 (with two members), CYP5493, CYP53, CYP5494, and CYP5495 (Table 1).

The functions of the proteins CrtS (carotenoid biosynthesis), Cyp61 and Cyp51 (ergosterol biosynthesis) were described in previous studies [17, 20,21]. To determine the possible functions of the other ten P450s of $X$. dendrorhous, a phylogenetic tree was constructed using all the $X$. dendrorhous $\mathrm{P} 450$ sequences and the amino acid sequences of 67 previously functionally characterized P450 proteins obtained from the Fungal Cytochrome P450 database (http://p450.riceblast.snu.ac.kr/ char_p450.php) and other works [9]. Protein multiple alignment was done with the online tool Clustal Omega, and the phylogenetic tree was constructed using the neighbor-joining statistical method with the Simple Phylogeny tool using the default parameters. The tree, originally in the Newick format, was visualized with MEGA 6.0 software [37], and the resulting tree is presented in Fig. 3.

In general, the thirteen P450s of X. dendrorhous could be separated into seven groups. In the first two groups, the $X$. dendrorhous Cyp51 and Cyp61 proteins grouped together with described Cyp51 and Cyp61 from other microorganisms, respectively. This was expected as the involvement of both enzymes in sterol biosynthesis has been extensively characterized in different organisms, including $X$. dendrorhous $[20,21]$. A third group contained Cyp5494A1 of X. dendrorhous, which was grouped apart from the other P450s of this yeast, and whose closest relatives are the "alk" proteins of Yarrowia lipolytica and different Candida species. This observation is very interesting, because the $A L K$ genes from different organisms have been studied due to their importance in the assimilation of $n$-alkanes and fatty acids as well as their participation in xenobiotic metabolism $[9,54]$. A fourth group included the CrtS, Cyp5139P1 and Cyp5139R1 proteins of $X$. dendrorhous, which were considerably distant from any other of the characterized fungal P450s. In this group, the $\mathrm{CrtS}$ protein has a known function in the astaxanthin biosynthesis. Based on the high identity percentage between Cyp5139P1 and CrtS (47\% identity, 65\% similitude), it is plausible that Cyp5139P1 could have a related function. Since Cyp5139P1 and Cyp5139R1 (this last one showed 35\% identity and 54\% similitude with CrtS) did not show similarity with any other functionally characterized enzyme, experimental studies are required to suggest their potential function. A fifth group included Cyp5491A1, Cyp5492A1, Cyp5493A1, Cyp5495A1 and Cyp5492A2 from $X$. dendrorhous, of which Cyp5492A1 and Cyp5492A2 were the most closely related, belonging to the same P450 family. In this case, no functionally characterized P450s from other fungi were related to these proteins, so it was not possible to suggest a potential function for this group of P450s, opening the opportunity to find new, undescribed functions for these proteins. In a sixth group, Cyp5490A1 of $X$. dendrorhous grouped with the proteins encoded by the fum6 and CYP505 genes of Fusarium oxysporum, which are involved in secondary metabolism reactions regarding the synthesis of the mycotoxin fumonisin and the $\omega-1$ to $\omega-3$ carbon hydroxylation of fatty acids, respectively $[9,55,56]$. Regarding fatty acid hydroxylation, the microbial production of hydroxyl fatty acids is of commercial interest because these compounds are widely used in the chemical, food, and cosmetic industries as starting materials for the synthesis of polymers and as additives for the manufacture of lubricants, emulsifiers, and stabilizers. Additionally, they have antibiotic, anti-inflammatory, and anticancer activities with potential medicinal uses [57]. In this sense, the function of Cyp5490A1 could be of great interest if it is related to the functions of the proteins encoded by fum6 or CYP505. Overall, the results suggest that Cyp5490A1 may participate in secondary metabolism. In the last group, the protein Cyp53C10 of $X$. dendrorhous grouped together with the proteins encoded by the genes $b z u A$ and $b p h$ (benzoate para-hydroxylase) of Aspergillus nidulans and Aspergillus niger, respectively. The $b z u A$ gene of $A$. nidulans encodes a protein orthologous to the benzoate parahydroxylase-encoding gene $b p h$ of $A$. niger; in both cases, 


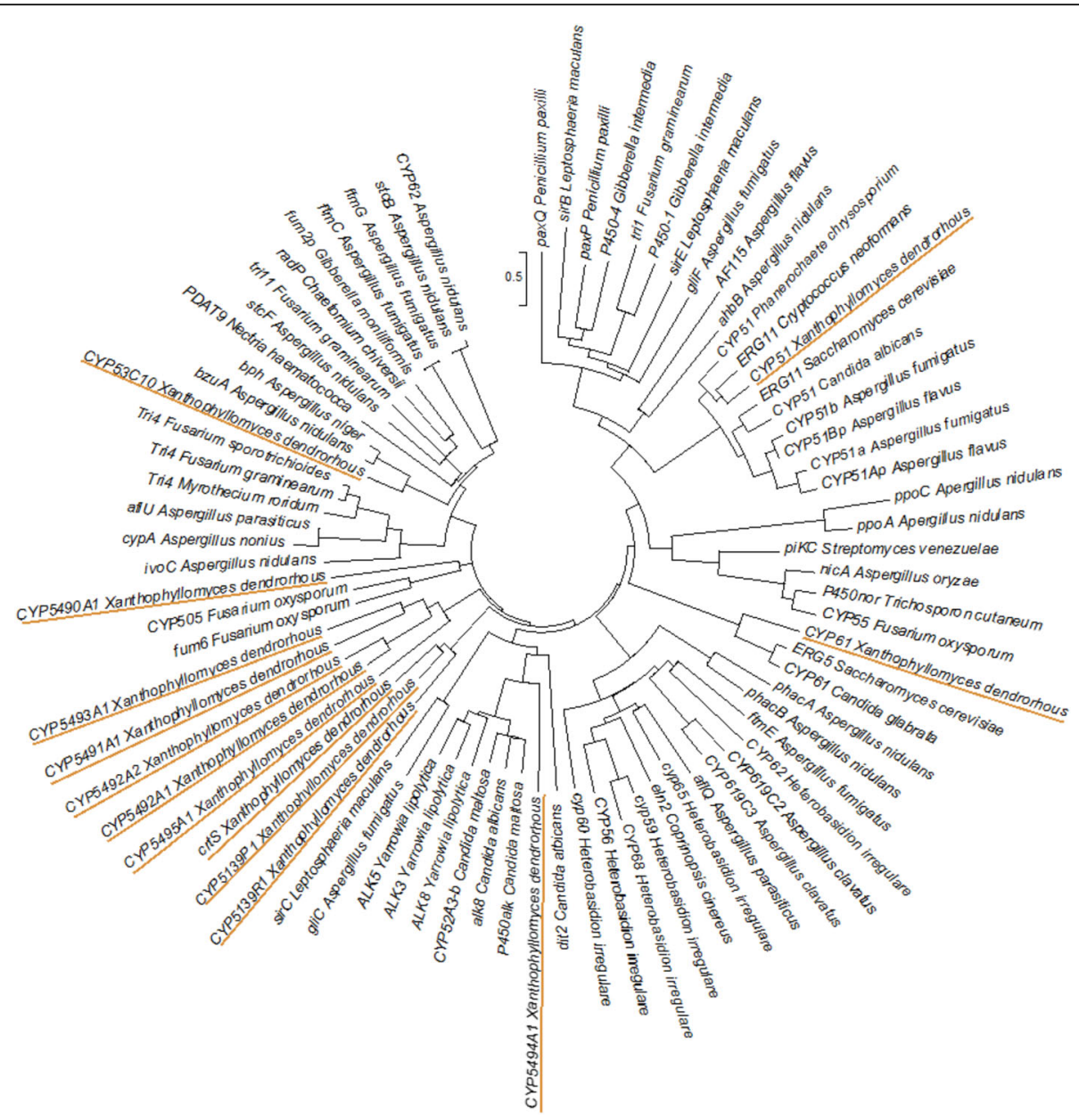

Fig. 3 Phylogenetic analysis tree of all X. dendrorhous P450s and other fungal P450s. The phylogenetic tree was created with the Simple Phylogeny tool using the neighbor-joining method with the amino acid sequences of all the P450s from $X$. dendrorhous (underlined) and 67 functionally characterized P450s from other organisms. In the tree, the names of the P450-encoding genes are detailed, followed by the organism of origin. The accession numbers of the sequences are indicated in the Methods section

the encoded enzymes participate in primary and xenobiotic metabolism (benzamide and benzoate degradation) $[58,59]$. This may be of great interest as Cyp53C10 of $X$. dendrorhous could be involved in the degradation of aromatic xenobiotic compounds such as benzoate and benzamide.

Finally, the phylogenetic analysis separated the thirteen P450s of $X$. dendrorhous into groups revealing the potential functions of these recently discovered genes, ranging from primary functions, such as sterols biosynthesis, to secondary and xenobiotic metabolism, including reactions of biotechnological interest.

\section{Conclusions}

The present study reveals that the carotenogenic yeast $X$. dendrorhous possesses thirteen P450-encoding genes, whose products may be involved in primary and secondary metabolism reactions. In the case of CrtS, Cyp51 and Cyp61, their functions in carotenogenesis and sterol synthesis, respectively, have been previously described. The possible functions of the other identified P450s include reactions involved in primary and xenobiotic metabolism such as fatty acid hydroxylation and aromatic compound degradation. These findings lay a foundation for future experiments to elucidate the role of the different P450s in $X$. dendrorhous and the possibility of discovering new functions and potential applications for this diverse group of enzymes.

\section{Additional files}

Additional file 1: Figure S1. Analysis of secondary structure elements in P450 proteins from $X$. dendrorhous. Characteristics conserved secondary elements of P450 proteins are shown along the thirteen P450 proteins of $X$. dendrorhous. Alpha helices ( $h$ ) and beta-strands (b) are represented as orange cylinders and blue arrows, respectively. The transmembrane region (TM) and conserved regions such as the "meander loop" and the "Cys pocket" are also highlighted. The locations of the conserved motifs AGxDTT, ExxR, PER and CxG are shown along the proteins. Residues 
potentially involved in the interaction between P450 and CrtR are indicated in red. (JPEG $1040 \mathrm{~kb}$ )

Additional file 2: Figure S2. Multiple alignment of the region from helix aC to helix aD of all X. dendrorhous P450s. An alignment was made using Geneious v.10 software, and residues were colored by polarity. Asterisks indicate the most conserved positively charged residues. (JPEG $344 \mathrm{~kb})$

\section{Abbreviations}

CPR: Cytochrome P450 reductase; FAD: Flavin adenine dinucleotide; FMN: Flavin mononucleotide; MMv: Vogel minimum medium; NADPH: Nicotinamide adenine dinucleotide phosphate; OBD: Oxygen-binding domain; ORF: Open reading frame; P450 or CYP: Cytochrome P450; pl: Isoelectric point; RPKM: Reads per kilobase per million mapped reads

\section{Acknowledgements}

None.

\section{Funding}

FONDECYT 1160202 financially supported this work.

\section{Availability of data and materials}

All data generated or analyzed during this study are included in this published article and its supplementary information files.

\section{Authors' contributions}

PC and JA carried out the bioinformatic analyses (gene identification, protein sequence analysis and phylogenetic analysis) and interpreted the results. AMG helped with the transcriptomic and promoter sequence analysis. DN named all genes following the standard P450 nomenclature. MSG helped defining P450 gene structure. MB and VC contributed to the study design and analysis of the results. JA conceived the study and participated in the experimental design and coordination. PC and JA drafted the manuscript. All authors read and approved the final manuscript.

\section{Ethics approval and consent to participate}

Not applicable.

\section{Consent for publication}

Not applicable.

\section{Competing interests}

All the authors declare that they have no competing interests.

\section{Publisher's Note}

Springer Nature remains neutral with regard to jurisdictional claims in published maps and institutional affiliations.

\section{Author details}

'Departamento de Ciencias Ecológicas y Centro de Biotecnología, Facultad de Ciencias, Universidad de Chile, Las Palmeras 3425, Casilla 653, Santiago, Chile. ${ }^{2}$ Department of Microbiology, Immunology and Biochemistry, University of Tennessee Health Science Center, Memphis, TN 38163, USA.

Received: 29 March 2017 Accepted: 13 July 2017

Published online: 19 July 2017

\section{References}

1. Zhang H, Im SC, Waskell L. Cytochrome b5 increases the rate of product formation by cytochrome P450 2B4 and competes with cytochrome P450 reductase for a binding site on cytochrome P450 2B4. J Biol Chem. 2007;282:29766-76.

2. Degtyarenko KN, Archakov Al. Molecular evolution of P450 superfamily and P450-containing monooxygenase systems. FEBS Lett. 1993;332:1-8.

3. Bernhardt R. Cytochromes P450 as versatile biocatalysts. J Biotechnol. 2006;124:128-45.

4. Estabrook RW. A passion for P450s (rememberances of the early history of research on cytochrome P450). Drug Metab Dispos. 2003;31:1461-73.
5. Park J, Lee S, Choi J, Ahn K, Park B, Park J, Kang S, Lee YH. Fungal cytochrome P450 database. BMC Genomics. 2008:9:402

6. Porter TD, Coon MJ. Cytochrome P-450. Multiplicity of isoforms, substrates, and catalytic and regulatory mechanisms. J Biol Chem. 1991;266:13469-72.

7. Kimmich N, Das A, Sevrioukova I, Meharenna Y, Sligar SG, Poulos TL. Electron transfer between cytochrome P450cin and its FMN-containing redox partner, cindoxin. J Biol Chem. 2007;282:27006-11.

8. Kelly SL, Kelly DE. Microbial cytochromes P450: biodiversity and biotechnology. Where do cytochromes P450 come from, what do they do and what can they do for us. Philos Trans R Soc Lond Ser B Biol Sci. 2013:368:20120476.

9. Črešnar B, Petrič Š. Cytochrome P450 enzymes in the fungal kingdom. Biochim Biophys Acta. 2011;1814:29-35.

10. McLean KJ, Sabri M, Marshall KR, Lawson RJ, Lewis DG, Clift D, Balding PR, Dunford AJ, Warman AJ, McVey JP. Biodiversity of cytochrome P450 redox systems. Biochem Soc T. 2005;33:796-801.

11. Munro AW, Girvan HM, McLean KJ. Cytochrome P450 - redox partner fusion enzymes. Biochim Biophys Acta. 2007;1770:345-59

12. Lah L, Krasevec N, Trontelj P, Komel R. High diversity and complex evolution of fungal cytochrome P450 reductase: cytochrome P450 systems. Fungal Genet Biol. 2008:45:446-58.

13. Nelson DR. The cytochrome P450 homepage. Hum Genomics. 2009;325:68-74.

14. Syed K, Nelson DR, Riley R, Yadav JS. Genomewide annotation and comparative genomics of cytochrome P450 monooxygenases (P450s) in the polypore species Bjerkandera adusta, Ganoderma sp and Phlebia brevispora. Mycologia. 2013;105:1445-55.

15. Hoffmeister D, Keller NP. Natural products of filamentous fungi: enzymes, genes, and their regulation. Nat Prod Rep. 2007;24:393-416.

16. Ojima K, Breitenbach J, Visser H, Setoguchi $Y$, Tabata K, Hoshino T, van den Berg J, Sandmann G. Cloning of the astaxanthin synthase gene from Xanthophyllomyces dendrorhous (Phaffia rhodozyma) and its assignment as a B-carotene 3-hydroxylase/4-ketolase. Mol Gen Genomics. 2006;275:148-58.

17. Alvarez V, Rodriguez-Saiz M, de la Fuente JL, Gudina EJ, Godio RP, Martin JF, Barredo JL. The crtS gene of Xanthophyllomyces dendrorhous encodes a novel cytochrome-P450 hydroxylase involved in the conversion of beta-carotene into astaxanthin and other xanthophylls. Fungal Genet Biol. 2006;43:261-72.

18. Alcaíno J, Barahona S, Carmona M, Lozano C, Marcoleta A, Niklitschek M, Sepulveda D, Baeza M, Cifuentes V. Cloning of the cytochrome P450 reductase $(c r t R)$ gene and its involvement in the astaxanthin biosynthesis of Xanthophyllomyces dendrorhous. BMC Microbiol. 2008:8:169.

19. Ukibe K, Hashida K, Yoshida N, Takagi H. Metabolic engineering of Saccharomyces cerevisiae for astaxanthin production and oxidative stress tolerance. Appl Environ Microbiol. 2009;75:7205-11.

20. Loto I, Gutierrez MS, Barahona S, Sepulveda D, Martinez-Moya P, Baeza M, Cifuentes $V$, Alcaíno J. Enhancement of carotenoid production by disrupting the C22-sterol desaturase gene (CYP61) in Xanthophyllomyces dendrorhous. BMC Microbiol. 2012:12:235.

21. Leiva K, Werner N, Sepulveda D, Barahona S, Baeza M, Cifuentes V, Alcaíno J. Identification and functional characterization of the CYP51 gene from the yeast Xanthophyllomyces dendrorhous that is involved in ergosterol biosynthesis. BMC Microbiol. 2015;15:89.

22. Chen W, Lee MK, Jefcoate C, Kim SC, Chen F, Yu JH. Fungal cytochrome P450 monooxygenases: their distribution, structure, functions, family expansion, and evolutionary origin. Genome Biol Evol. 2014;6:1620-34

23. Retamales $P$, Hermosilla $G$, Leon R, Martinez C, Jimenez A, Cifuentes V. Development of the sexual reproductive cycle of Xanthophyllomyces dendrorhous. J Microbiol Methods. 2002:48:87-93.

24. Wozniak A, Lozano C, Barahona S, Niklitschek M, Marcoleta A, Alcaíno J, Sepulveda D, Baeza M, Cifuentes V. Differential carotenoid production and gene expression in Xanthophyllomyces dendrorhous grown in a nonfermentable carbon source. FEMS Yeast Res. 2011;11:252-62.

25. Cifuentes $V$, Hermosilla $G$, Martinez $C$, Leon R, Pincheira G, Jimenez A. Genetics and electrophoretic karyotyping of wild-type and astaxanthin mutant strains of Phaffia rhodozyma. Antonie Van Leeuwenhoek. 1997;72:111-7.

26. Lodato P, Alcaíno J, Barahona S, Retamales P, Jimenez A, Cifuentes V. Study of the expression of carotenoid biosynthesis genes in wild-type and deregulated strains of Xanthophyllomyces dendrorhous (ex:: Phaffia rhodozyma). Biol Res. 2004;37:83-93.

27. Chomczynski P, Sacchi N. The single-step method of RNA isolation by acid guanidinium thiocyanate-phenol-chloroform extraction: twenty-something years on. Nat Protoc. 2006;1:581-5. 
28. Sambrook J, Russell DW. Molecular cloning: a laboratory manual 3rd ed. NY: Cold Spring Harbor Laboratory Press, Cold Spring Harbor; 2001.

29. Baeza M, Alcaíno J, Barahona S, Sepúlveda D, Cifuentes V. Codon usage and codon context bias in Xanthophyllomyces dendrorhous. BMC Genomics. 2015;16:293

30. Hofmann K, Stoffel W. TMbase - a database of membrane spanning protein segments. Biol Chem Hoppe Seyler. 1993;374:166.

31. Drozdetskiy A, Cole C, Procter J, Barton GJ. JPred4: a protein secondary structure prediction server. Nucleic Acids Res. 2015;43:W389-94.

32. Arnold K, Bordoli L, Kopp J, Schwede T. The SWISS-MODEL workspace: a web-based environment for protein structure homology modelling. Bioinformatics. 2006;22:195-201.

33. Biasini M, Bienert S, Waterhouse A, Arnold K, Studer G, Schmidt T, Kiefer F, Gallo Cassarino T, Bertoni M, Bordoli L, Schwede T. SWISS-MODEL: modelling protein tertiary and quaternary structure using evolutionary information. Nucleic Acids Res. 2014;42:W252-8.

34. Kel AE. MATCHTM: a tool for searching transcription factor binding sites in DNA sequences. Nucleic Acids Res. 2003;31:3576-9.

35. Tsunoda T, Takagi T. Estimating transcription factor bindability on DNA. Bioinformatics. 1999;15:622-30.

36. Mathelier A, Fornes O, Arenillas DJ, Chen CY, Denay G, Lee J, Shi W, Shyr C, Tan G, Worsley-Hunt R, Zhang AW, Parcy F, Lenhard B, Sandelin A, Wasserman WW. JASPAR 2016: a major expansion and update of the openaccess database of transcription factor binding profiles. Nucleic Acids Res. 2016:44:D110-5.

37. Tamura K, Stecher G, Peterson D, Filipski A, Kumar S. MEGA6: molecular evolutionary genetics analysis version 6.0. Mol Biol Evol. 2013;30:2725-9.

38. Sharma R, Gassel S, Steiger S, Xia X, Bauer R, Sandmann G, Thines M. The genome of the basal agaricomycete Xanthophyllomyces dendrorhous provides insights into the organization of its acetyl-CoA derived pathways and the evolution of Agaricomycotina. BMC Genomics. 2015;16:233.

39. Mortazavi A, Williams BA, McCue K, Schaeffer L, Wold B. Mapping and quantifying mammalian transcriptomes by RNA-Seq. Nat Methods. 2008;5:621-8.

40. van den Brink JM, Punt PJ, van Gorcom RFM, van Den Hondel CAMJJ. Regulation of expression of the Aspergillus niger benzoate para-hydroxylase cytochrome P450 system. Mol Gen Genet. 2000;263:601-9.

41. Stiborová M, Suchá V, Miksanova M, Páca J, Páca J. Hydroxylation of phenol to catechol by Candida tropicalis: involvement of cytochrome P450. Gen Physiol Biophys. 2003;22:167-80.

42. Lepesheva GI, Waterman MR. CYP51 - the omnipotent P450. Mol Cell Endocrinol. 2004;215:165-70.

43. Lepesheva GI, Waterman MR. Sterol 14a-demethylase cytochrome P450 (CYP51), a P450 in all biological kingdoms. Biochim Biophys Acta. 1770;2007:467-77.

44. Todd BL, Stewart EV, Burg JS, Hughes AL, Espenshade PJ. Sterol regulatory element binding protein is a principal regulator of anaerobic gene expression in fission yeast. Mol Cell Biol. 2006;26:2817-31.

45. Hasemann CA, Kurumbail RG, Boddupalli SS, Peterson JA, Deisenhofer J. Structure and function of cytochromes P450: a comparative analysis of three crystal structures. Structure. 1995;3:41-62.

46. Hernández-Martínez F, Briones-Roblero Cl, Nelson DR, Rivera-Orduña FN, Zúñiga G. Cytochrome P450 complement (CYPome) of Candida oregonensis, a gut-associated yeast of bark beetle, Dendroctonus rhizophagus. Fungal Biol. 2016;120:1077-89.

47. Syed K, Mashele SS. Comparative analysis of P450 signature motifs EXXR and CXG in the large and diverse kingdom of fungi: identification of evolutionarily conserved amino acid patterns characteristic of P450 family. PLoS One. 2014;9:e95616.

48. Kelly DE, Krasevec N, Mullins J, Nelson DR. The CYPome (Cytochrome P450 complement) of Aspergillus nidulans. Fungal Genet Biol. 2009;46:S53-61.

49. Mestres J. Structure conservation in cytochromes P450. Proteins. 2005;58:596-609.

50. Peterson JA, Graham SE. A close family resemblance: the importance of structure in understanding cytochromes P450. Structure. 1998;6:1079-85.

51. Sirim D, Widmann M, Wagner F, Pleiss J. Prediction and analysis of the modular structure of cytochrome P450 monooxygenases. BMC Struct Biol. 2010;10:34.

52. Alcaíno J, Fuentealba M, Cabrera R, Baeza M, Cifuentes V. Modeling the interfacial interactions between CrtS and CrtR from Xanthophyllomyces dendrorhous, a P450 system involved in astaxanthin production. J Agric Food Chem. 2012;60:8640-7.

53. Gutiérrez MS, Rojas MC, Sepúlveda D, Baeza M, Cifuentes V, Alcaíno J. Molecular characterization and functional analysis of cytochrome b5 reductase (CBR) encoding genes from the carotenogenic yeast Xanthophyllomyces dendrorhous. PLoS One. 2015;10:e0140424.

54. Iwama R, Kobayashi S, Ishimaru C, Ohta A, Horiuchi H, Fukuda R. Functional roles and substrate specificities of twelve cytochromes P450 belonging to CYP52 family in n-alkane assimilating yeast Yarrowia lipolytica. Fungal Genet Biol. 2016;91:43-54.

55. Proctor RH, Busman $M$, Seo JA, Lee YW, Plattner RD. A fumonisin biosynthetic gene cluster in Fusarium oxysporum strain 0-1890 and the genetic basis for B versus C fumonisin production. Fungal Genet Biol. 2008;45:1016-26.

56. Uhlig S, Busman M, Shane DS, Rønning H, Rise F, Proctor R. Identification of early fumonisin biosynthetic intermediates by inactivation of the FUM6 gene in Fusarium verticillioides. J Agric Food Chem. 2012;60:10293-301.

57. Kim KR, Oh DK. Production of hydroxy fatty acids by microbial fatty acidhydroxylation enzymes. Biotechnol Adv. 2013;31:1473-85.

58. Faber BW, van Gorcom RF, Duine JA. Purification and characterization of benzoate-para-hydroxylase, a cytochrome P450 (CYP53A1), from Aspergillus niger. Arch Biochem Biophys. 2001;394:245-54.

59. Fraser JA, Davis MA, Hynes MJ. The genes gmdA, encoding an amidase, and bzuA, encoding a cytochrome P450, are required for benzamide utilization in Aspergillus nidulans. Fungal Genet Biol. 2002;35:135-46.

\section{Submit your next manuscript to BioMed Central and we will help you at every step:}

- We accept pre-submission inquiries

- Our selector tool helps you to find the most relevant journal

- We provide round the clock customer support

- Convenient online submission

- Thorough peer review

- Inclusion in PubMed and all major indexing services

- Maximum visibility for your research

Submit your manuscript at www.biomedcentral.com/submit
) Biomed Central 